\title{
Integrating Healthy Eating and Active Living through Knowledge Translation \\ And Inter-Disciplinary Collaborations
}

\author{
Teresa L. Penbrooke ${ }^{1}$ \\ ${ }^{1}$ GP RED, www.gpred.org, and GreenPlay LLC, Louisville, Colorado, USA
}

Keywords: knowledge translation, preventive health, systems thinking, academic institutions, non-profits, professionals, recreation, active living 
It is an honor to introduce this Special Edition of the Journal of Healthy Eating and Active Living (JHEAL), as a partner journal for the 2021 Active Living Conference $(A L C)$, which was held as a virtual education and engagement event online on April $6^{\text {th }}$ through 9th, 2021. As part of an alliance, the editors and reviewers of JHEAL worked to produce this special issue devoted to research, practice, and policy on active living presented at the conference. Some of the top scoring proposals selected for presentation at the conference were invited to submit papers for inclusion. Authors of both research and practice/policy focused abstracts were asked whether they wish to be considered. Many of the papers address issues related to the 2021 ALC theme, Now More than Ever: Active Living for All. Papers for this issue were invited for a full manuscript or a brief report, depending on the complexity of the topic.

The JHEAL/GP RED alliance emerged from the collaboration and connections across multiple organizations. As explained by Dr. James F. Sallis in the inaugural issue of JHEAL (Sallis, 2020), JHEAL evolved in part from a paradigm shift promoted by Active Living Research (ALR - www.activelivingresearch.org), along with previous and ongoing healthy eating and active living (HEAL) research. When Dr. Sallis retired from his role as founder and long-time key creator of ALR and the resultant conference (ALC), and initial funding changes occurred, there was a need to find a way to continue this good work and annual gatherings in a sustainable way. Other conference organizers worked with ALR staff for a few years to plan the meeting, and then in the summer of 2020, Dr. Sallis and key ALC supporters enthusiastically agreed that the ALC and research archives could be transferred to be under the organizational and fiscal agency of GP RED (www.gpred.org). This was primarily to find a permanent, sustainable home for the annual ALC and to allow for a lower cost, effective, and nimble way to allow for ongoing convening of the researchers, practitioners, educators, and other allied professionals. The goal was to continue to identify and share the important growing body of work related to active living.

GP RED is a board-governed 501(c)(3) non-profit organization founded in 2009 that works to help the professionals who help communities thrive through targeted support of connecting, convening, and collaborating in the realms of preventive health, parks and recreation $(\mathrm{P} \& \mathrm{R})$, land management, and allied disciplines. A key long-time staff person for ALC, Amanda Walker, became an employee of GP RED to continue as the Research and Education Manager for the ALC.

ALC and JHEAL both align well with the work of GP RED's Healthy Communities Research Group, the SHIFT Summit (a gathering focused on the intersection of access to nature, conservation, and preventive health), and other related initiatives. GP RED's target audience is similar to the target audience for JHEAL with additional focus on practice, equity and inclusion, and inter-disciplinary relationships. GP RED supports frequent contacts with over 20,000 educators, researchers, practice, and related professionals, and works to provide the management, fiscal oversite, meeting management, marketing, and technological support that are needed to continue the good work of ALC and the growing group of thought leaders in this combined realm.

GP RED, ALC, and allied professionals interested in JHEAL topics cannot do this good work alone. GP RED supports a vision of collaboration that helps to fill the gaps and minimize duplication of efforts across education, research, and related industries, translating knowledge into practice, identifying emerging trends, and serving as an incubator for innovative solutions. GP RED supports information exchange from research to practice (and vice versa) through systems thinking approaches, especially as adapted for parks and recreation (CDC, 2014; Penbrooke, 2017) as shown in Figure 1. 
Figure 1

Adapted Knowledge to Action (K2A) Framework

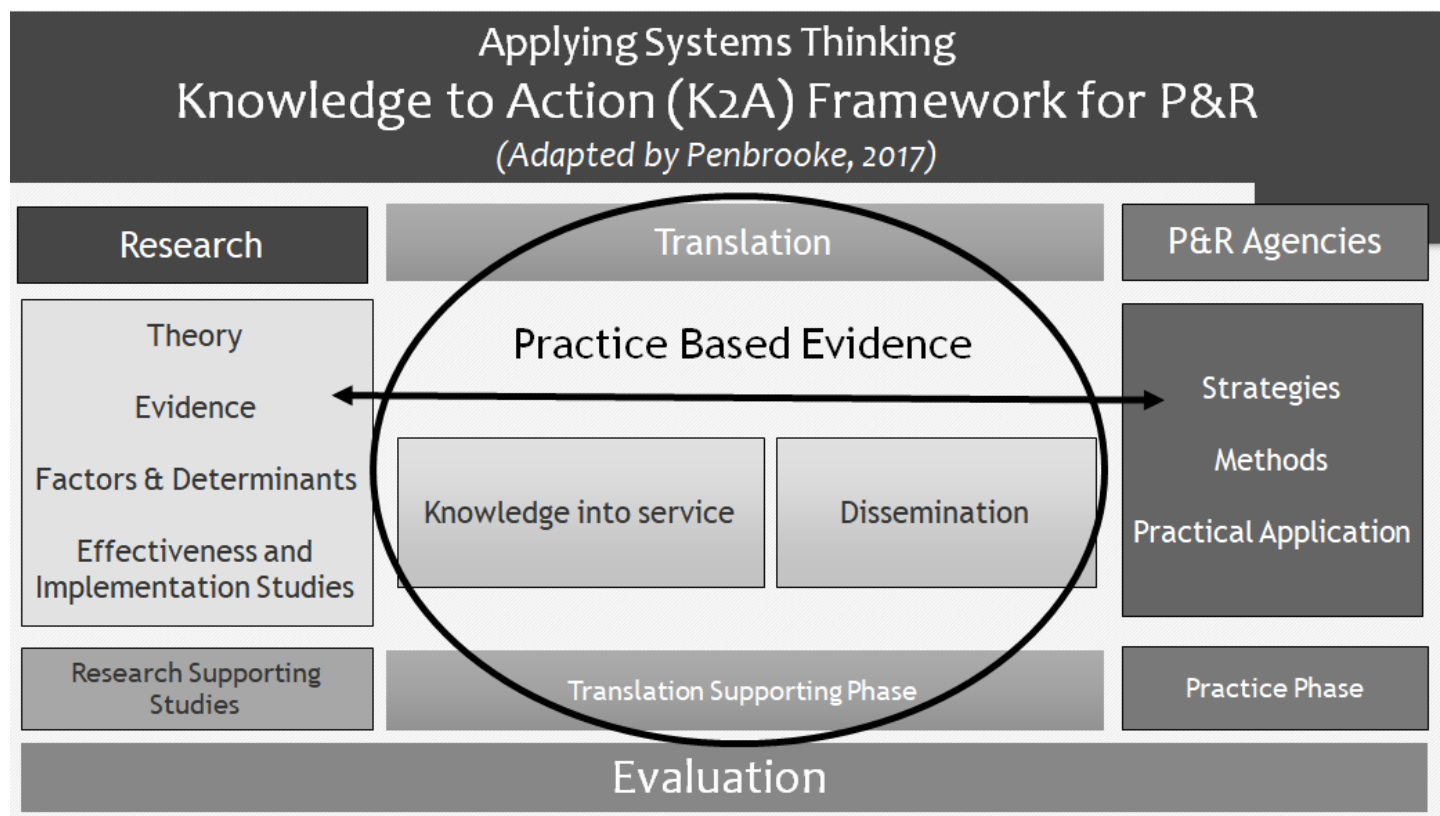

It is no secret that these are challenging times for academic institutions, non-profits, and related businesses and governmental organizations. GP RED and ALC have struggled since inception to find strong, sustainable revenue and income sources. The lack of dedicated funding has constrained staffing capacity and growth. Primary goals for alliances like this are to increase awareness for all entities, build financial sustainability, increase staffing and funding, along with supporting ongoing good work through research and application to practice.

As an international, open-access, quarterly, peerreviewed journal focused on publishing high-quality studies in the areas of active living, active transportation, healthy eating, and the relationship between the natural, built, virtual and social environments, JHEAL hopes to increase the reach and awareness for these ALC topics. JHEAL readers are invited to submit to and attend future ALC gatherings - https://www.gpred.org/activelivingconference and to submit to layperson-language, practice-focused summaries that can be distributed as shorter GP RED Research Briefs (www.gpred.org/research-briefs). GP RED maintains over 25 active alliances with various publications, associations, universities, and organizations, and invites the opportunity for future collaborations. The hope is that that together we can all continue to expand and grow our impact.

Correspondence should be addressed to:

Teresa L. Penbrooke

1021 E. S. Boulder Rd., Ste. N

Louisville, CO 80027

303-870-3884

teresap@gpred.org or teresap@greenplayllc.com

(1) Teresa L. Penbrooke: 0000-0002-4705-7472

Creative Commons License:

This work is licensed under a Creative Commons Attribution-Noncommercial 4.0 International License (CC BY-NC 4.0).

Suggested Citation (APA 7th edition):

Penbrooke, T.L. (2021). Integrating healthy eating and active living through knowledge translation and interdisciplinary collaborations. Journal of Healthy Eating and Active Living, 1(4), 161-164. 
Journal of Healthy Eating and Active Living

2021, Vol. 1, No. 4, pgs. 161-164

\section{References}

Centers for Disease Control and Prevention. Applying the Knowledge to Action (K2A) Framework: Questions to Guide Planning. Atlanta, GA: Centers for Disease Control and Prevention, US Dept of Health and Human Services; 2014. Available at http://intranet.cdc.gov/nccdphp/home/WGOT/tools/index.html

Penbrooke, T.L. (2017). Local parks and recreation agencies use of systems thinking to address preventive public health factors. (Doctoral Dissertation). North Carolina State University, Raleigh, NC.

https://repository.lib.ncsu.edu/handle/1840.20/34758.

Sallis, J. F. (2020). JHEAL: What does it mean to initiate a new journal? Journal of Healthy Eating and Active Living, 1(1), 1-2. 